\title{
CÁLCULO DAS VARIÁVEIS NA DESIDRATAÇ̃̃o OSMÓTICA DE MANGA CV. TOMMY ATKINS
}

\author{
Calculation of the variables in dehydration osmotic of the mangoes cv. Tommy Atkins
}

\author{
Nelisa Sita Pires Picolotto Martim¹, Nina Waszczynskyj², Maria Lucia Masson ${ }^{3}$
}

\begin{abstract}
RESUMO
A desidratação osmótica é usada para a remoção da água, de frutas imersas em solução aquosa. A qualidade da desidratação osmótica depende de diferentes fatores como: concentração, agente desidratante, temperatura, tempo de imersão, natureza das frutas e a área de superfície exposta à troca osmótica. A parede celular e o tipo de açúcar utilizado como agente osmótico podem influenciar no fenômeno de transferência de massa. A eficiência do processo de desidratação osmótica pode ser quantificada pelos valores de perda de peso e incorporação de sólidos. As mangas foram cortadas em cubos de $1 \mathrm{~cm}$ de lado e levadas para os tratamentos com $50 \%$ sacarose (SAC), 50\% sorbitol (SOR) e 50\% estévia comercial (STV) acrescido de $1 \%$ de cloreto de cálcio e o pH corrigido em 4,0 com o uso de ácido cítrico, por duas horas a $50^{\circ} \mathrm{C}$. A proporção fruta:solução desidratante utilizada em todos os tratamentos foi de 1:3 em massa. O tratamento utilizando solução composta com estévia não se mostrou eficiente por causa do baixo ganho de sólidos e reduzida perda de água. A maior perda de água e uma maior perda de peso da manga durante a desidratação osmótica foram observadas para o tratamento com sorbitol, enquanto o maior ganho de sólidos foi obtido no tratamento com sacarose.
\end{abstract}

Termos para indexação: Manga, desidratação osmótica, sacarose, sorbitol, estévia.

\section{ABSTRACT}

The osmotic dehydration is used for water removal from immersed fruits in watery solution. The quality of the osmotic dehydration depends on different factors as concentration, dehydrating agent, temperature, time of immersion, nature of fruits and the surface displayed to the osmotic exchange. The cellular wall and the type of used sugar as osmotic agent can influence in the phenomenon of mass transference. The efficiency of the process of osmotic dehydration can be quantified by the values of loss of weight and solid incorporation. The mangoes had been cut in cubes of $1 \mathrm{~cm}$ of side and taken for the treatments with $50 \%$ sucrose (SAC), 50\% sorbitol (SOR) and 50\% commercial estévia (STV) increased of $1 \%$ of calcium chloride and pH corrected in 4,0 with the acid use of citric, for two hours in $50^{\circ} \mathrm{C}$. The ratio fruit:solution used in all the treatments was of 1:3. The treatment using composed solution with estévia did not show to be efficient due to the low solid profit and reduced loss of water A bigger loss of water and a bigger loss of weight of the mango during the osmotic dehydration had been observed for the treatment with sorbitol, while the biggest solid profit was gotten in the treatment with sucrose.

Index terms: Mango, osmotic dehydration, sucrose, sorbitol, estévia.

(Recebido em 17 de outubro de 2006 e aprovado em 19 de junho de 2007)

\section{INTRODUÇÃO}

A desidratação osmótica é uma técnica usada para a concentração por remoção da água, de frutas imersas em solução aquosa. É utilizada como prétratamento, seguida por tratamentos como secagem com ar aquecido, secagem a vácuo, congelamento entre outros (ANGELINI, 2002; ANTONIO, 2002; MIZRAHI et al., 2001).

A qualidade da desidratação osmótica depende de fatores como a concentração, o tipo de agente desidratante, a temperatura da solução, a pressão de trabalho, o tempo de imersão, a natureza das frutas e a área de superfície exposta à troca osmótica (MACCARTHY, 1986; SANTOS, 2003; TEDJO et al., 2002).

A perda de água durante a desidratação osmótica divide-se em duas etapas, no período inicial (aproximadamente 2 horas) ocorre alta taxa de remoção de água e em aproximadamente 30 minutos o elevado ganho de sólidos. Numa segunda etapa, de 2 a 6 horas ocorre um decréscimo da taxa de remoção de água (ANGELINI, 2002; ANTONIO, 2002; TORREGGIANI, 1993).

A parede celular e o tipo de açúcar utilizado como agente osmótico podem influenciar no fenômeno de transferência de massa. $\mathrm{O}$ uso de um agente osmótico com

\footnotetext{
Mestre em Tecnologia de Alimentos - Programa de Pós-Graduação em Tecnologia de Alimentos/TC-PPGTA - Universidade Federal do Paraná/UFPR Rua Bororrós, 1398, Portão - 81531-990 - Curitiba, PR - nemartim@gmail.com

${ }^{2}$ Doutora em Ciências - Programa de Pós-Graduação em Tecnologia de Alimentos/TC-PPGTA - Universidade Federal do Paraná/UFPR - Rua Francisco H. dos Santos, s/n, Jardim das Américas - Cx. P.19011 - 81531-990 - Curitiba, PR - ninawas@ufpr.br

${ }^{3}$ Doutora em Engenharia de Alimentos - Programa de Pós-Graduação em Tecnologia de Alimentos/TC-PPGTA - Universidade Federal do Paraná/UFPR Rua Francisco H. dos Santos, s/n, Jardim das Américas - Cx. P.19011 - 81531-990 - Curitiba, PR - masson@ufpr.br
} 
maior peso molecular pode provocar uma diminuição no ganho de sólidos e o aumento na perda de água, favorecendo a perda de massa. Sacarídeos de baixo peso molecular como a glicose, frutose e sorbitol favorecem o ganho de sólidos devido à alta taxa de penetração das moléculas do soluto (ANTONIO, 2002; TORREGGIANI, 1993).

A temperatura da desidratação osmótica tem uma importante influência na cinética, bem como na qualidade do produto final. $\mathrm{O}$ aumento da temperatura faz com que ocorra maior remoção de água e um decréscimo no tempo de tratamento (ANTONIO, 2002).

A eficiência do processo de desidratação osmótica pode ser quantificada pelos valores de perda de peso e incorporação de sólidos (ANGELINI, 2002; SANTOS, 2003).

O presente estudo foi realizado com o objetivo de determinar a perda de peso, ganho de sólidos e perda de água na desidratação osmótica de manga (Mangifera indica L. cv. Tommy Atkins.)

\section{MATERIALE MÉTODOS}

\section{Experimento}

As mangas in natura foram pesadas em balança semi-analítica. Após esta etapa as mangas foram lavadas em água clorada a 50ppm, para higienizar a casca da fruta.

Após descascadas, descaroçadas as frutas foram cortadas manualmente em cubos de $1 \mathrm{~cm}$ de lado e separados lotes para os tratamentos com $50 \%$ sacarose (SAC), 50\% sorbitol (SOR) e 50\% estévia comercial (STV).

Os cubos de manga foram imersos em solução desidratante a $50 \%(\mathrm{p} / \mathrm{p})$ previamente aquecida a $50^{\circ} \mathrm{C}$, acrescido de $1 \%$ de cloreto de cálcio e o pH corrigido em 4,0 com o uso de ácido cítrico.

Os tratamentos SAC, SOR e STV foram conduzidos à desidratação osmótica, por duas horas a $50^{\circ} \mathrm{C}$. O experimento de desidratação osmótica foi composto por sacos telados de poliéster com malha de $2 \mathrm{~mm}$, mantidos submersos com o auxilio de uma grade de aço inoxidável. A proporção fruta:solução desidratante utilizada em todos os tratamentos foi de 1:3 em massa.

\section{Métodos}

\section{Determinação de SST}

A porcentagem de sólidos solúveis totais das mangas in natura e desidratadas foi determinada utilizando-se um refratômetro de bancada RL3 - Polskie Zaklandy Optyczne S.A.com escala $0^{\circ}$ Brix a $90^{\circ}$ Brix $n^{\circ} 13.6 .1$ (INSTITUTO ADOLFO LUTZ, 1985). Os resultados, após correção em função da temperatura, foram expressos em graus Brix ( ${ }^{\circ}$ Brix).

\section{Determinação de umidade}

A determinação de umidade das amostras das mangas in natura e desidratadas foi quantificada pelo método de secagem em estufa a $105^{\circ} \mathrm{C}$ por 24 horas até massa constante conforme recomendado pelo IAL $\mathrm{n}^{\circ}$.4.5.1 (INSTITUTO ADOLFO LUTZ, 1985), os resultados foram expressos em porcentagem (\%).

\section{Perda de água (PA), ganho de sólidos (GS) e perda de $\operatorname{peso}\left(\mathbf{P}_{\mathbf{p}}\right)$}

Os percentuais de perda de água (PA), ganho de sólidos (GS) e perda de peso $\left(\mathrm{P}_{\mathrm{P}}\right)$, com base na massa inicial do material, no processo de desidratação osmótica podem ser obtidos através das equações 01, 02 e 03 (LIMA et al., 2004; SOUZA NETO et al., 2004):

$$
\begin{aligned}
& P A(\%)=\frac{\left(P_{0} \cdot U_{o}\right)-\left(P_{t} \cdot U_{t}\right)}{P_{0}} \\
& G S(\%)=\frac{\left(P_{t} \cdot B_{t}\right)-\left(P_{0} \cdot B_{o}\right)}{P_{0}} \\
& P_{P}(\%)=\frac{\left(P_{0} \cdot P_{t}\right)}{P_{0}} x 100
\end{aligned}
$$

Onde:

$\mathrm{P}_{0}=$ Massa da fruta no tempo $\mathrm{t}=0(\mathrm{~kg})$;

$\mathrm{P}_{\mathrm{t}}=$ Massa da fruta no tempo $\mathrm{t}(\mathrm{kg})$;

$\mathrm{U}_{0}=$ Umidade da fruta no tempo $\mathrm{t}=0(\mathrm{~s})$;

$\mathrm{U}_{\mathrm{t}}=$ Umidade da fruta no tempo $\mathrm{t}(\mathrm{s})$;

$\mathrm{B}_{0}={ }^{\circ}$ Brix da fruta no tempo $\mathrm{t}=0$ ( $\left.{ }^{\circ} \mathrm{Brix}\right)$;

$\mathrm{B}_{\mathrm{t}}={ }^{\circ} \mathrm{Brix}$ da fruta no tempo $\mathrm{t}\left({ }^{\mathrm{o}} \mathrm{Brix}\right)$.

\section{RESULTADOS E DISCUSSÃO}

\section{Perda de água}

Pela figura 01 ilustram-se os dados da perda de água $\left(\mathrm{P}_{\mathrm{A}}\right)$ para os tratamentos de desidratação osmótica utilizando soluções de sacarose (SAC), sorbitol (SOR) e estévia (STV), durante 2 (duas) horas de tratamento.

Ao analisarmos o gráfico indicado na figura 01, é observada uma perda de água ao final da desidratação osmótica (DO) de 33,46\% para o tratamento com sorbitol (SOR), de 28,16\% para o tratamento com sacarose (SAC) e de $0,86 \%$ para a solução composta com estévia $(\mathrm{p}<0.05)$.

$\mathrm{O}$ valor de perda de água para o tratamento com sacarose se situa no intervalo de $28 \%$ a $35 \%$ encontrado por Souza Neto et al. (2004) em seu estudo com manga, utilizando solução de sacarose $45^{\circ}-55^{\circ}$ Brix $/ 65^{\circ} \mathrm{C}$. Santos (2003) realizou um estudo com mangas e encontrou em média $36,66 \%$ de perda de água, sendo esse valor superior 
ao encontrado no presente estudo, e essa diferença pode ser atribuída, à diferença na concentração da solução (65 ${ }^{\circ}$ Brix) e à geometria da amostra (placas planas) utilizada por aquele autor.

Figura 01 - Perda de água $\left(\mathrm{P}_{\mathrm{A}}\right)$ para manga tratada osmoticamente com sacarose (SAC), sorbitol (SOR) e estévia (STV).

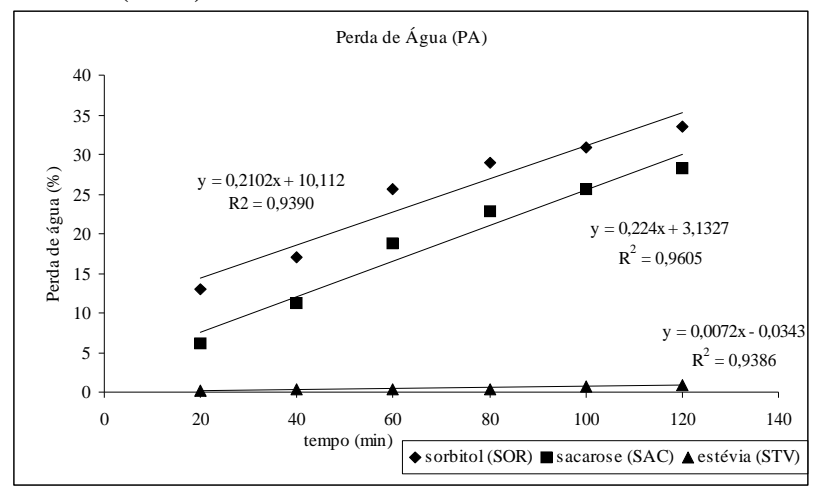

O valor encontrado, indicado na figura 01, para o tratamento com estévia $\left(\mathrm{PM}_{\text {estévia }}=804\right)$ mostra que esse agente osmótico não apresenta um bom resultado na remoção de água da manga, pois apresentou perda de água máxima igual a $0,86 \%$, este fato confirma que solutos de alto peso molecular não são eficientes na desidratação osmótica.

Pela figura 01 verifica-se que o tratamento com sorbitol apresenta perda de água significativamente $(\mathrm{p}<0,05)$ maior do que o tratamento com sacarose, este fato pode ser devido a diferença de massa molecular dos solutos $\left(\mathrm{PM}_{\text {sorbitol }}=182\right.$ e $\left.\mathrm{PM}_{\text {sacarose }}=342\right)$ indicando que o sorbitol tem maior facilidade de incorporação na fruta, e, sabendo-se que o sorbitol é um soluto higroscópico, verifica-se que este tem maior capacidade de desidratação da fruta.

\section{Ganho de sólidos}

A figura 02 ilustra os dados de ganho de sólidos (Gs) para os tratamentos de desidratação osmótica utilizando soluções de sacarose (SAC), sorbitol (SOR) e estévia (STV), durante 2 (duas) horas de tratamento.

Observa-se na figura 02 um ganho de sólidos ao final da desidratação osmótica (DO) de aproximadamente $9,93 \%$ para o tratamento com sacarose (SAC), de 6,31\% para o tratamento com sorbitol (SOR) e de $0,49 \%$ para a solução composta com estévia.

O valor de ganho de sólidos para o tratamento com sacarose está dentro da faixa (7\% a 10\%) encontrada por
Souza Neto et al. (2004) no estudo com manga osmoticamente desidratada com solução de sacarose de $45^{\circ}$ a $55^{\circ}$ Brix $/ 65^{\circ} \mathrm{C}$. Santos (2003) avaliou para a manga e encontrou em média $12 \%$ de ganho de sólidos, sendo esse valor superior ao encontrado no presente estudo. Esse fato pode ser explicado provavelmente pela geometria da amostra utilizada que eram fatias, ou seja placas planas o que facilita a incorporação de sólidos por causa da maior área de contato da manga com a solução desidratante.

Figura 02 - Ganho de sólidos $\left(\mathrm{G}_{\mathrm{S}}\right)$ para manga tratada osmóticamente com sacarose (SAC), sorbitol (SOR) e estévia (STV).

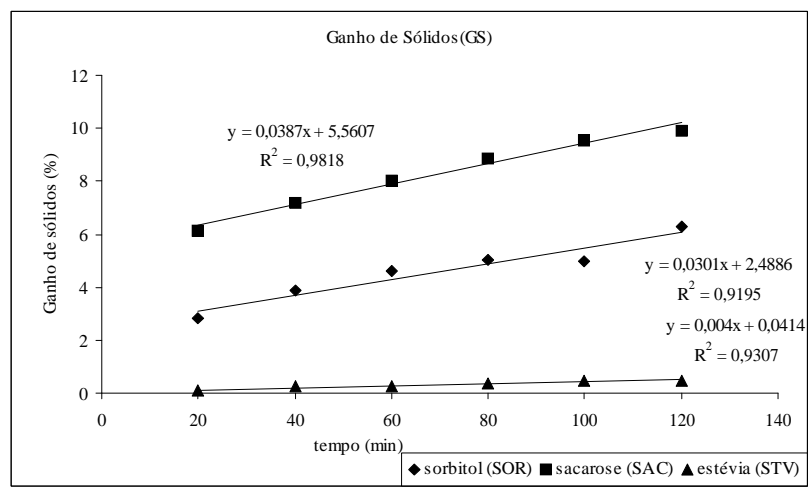

O valor encontrado para o tratamento com estévia mostra que esse agente osmótico não apresenta um bom resultado no ganho de sólidos da manga.

Pela figura 02 verifica-se que o tratamento osmótico com sacarose apresenta ganho significativo $(\mathrm{p}<0,05) \mathrm{de}$ sólidos se comparado ao tratamento com sorbitol, este fato fica evidenciado pelos valores obtidos com o tempo de $120 \mathrm{~min}$, de ganho no tratamento SAC de $9,93 \%$ e de $6,31 \%$ no tratamento SOR.

\section{Perda de peso}

A figura 03 indica os dados obtidos de perda de peso $\left(\mathrm{P}_{\mathrm{P}}\right)$ para os tratamentos estudados, durante 2 (duas) horas de processo de desidratação osmótica.

Observa-se uma perda de peso ao final da desidratação osmótica de aproximadamente $19,65 \%$ para o tratamento com sacarose, de $23,91 \%$ para o tratamento com sorbitol e de $0,1 \%$ para a solução composta com estévia. Sendo os tratamentos SAC e SOR com comportamentos de perda de peso bem similares, porém diferentes significativamente $(\mathrm{p}<0.05)$.

O valor de perda de peso para o tratamento osmótico com sacarose $(19,65 \%)$, se encontra dentro da faixa (18\% a $24 \%$ ) obtida por Souza Neto et al. (2004) e inferior ao 
encontrado por Santos (2003) onde este encontrou em média $28,5 \%$ de perda de peso. Esse fato pode ser explicado pela geometria (placas planas) da amostra utilizada, o que facilita a incorporação de sólidos e a perda de peso por causa da maior área de contato da fruta com a solução desidratante, fato também relatado por Sablani \& Rahman (2002) que afirmaram que a geometria da amostra influencia na cinética da desidratação osmótica.

Figura 03 - Perda de peso $\left(\mathrm{P}_{\mathrm{P}}\right)$ para manga tratada osmoticamente com sacarose (SAC), sorbitol (SOR) e stévia (STV).

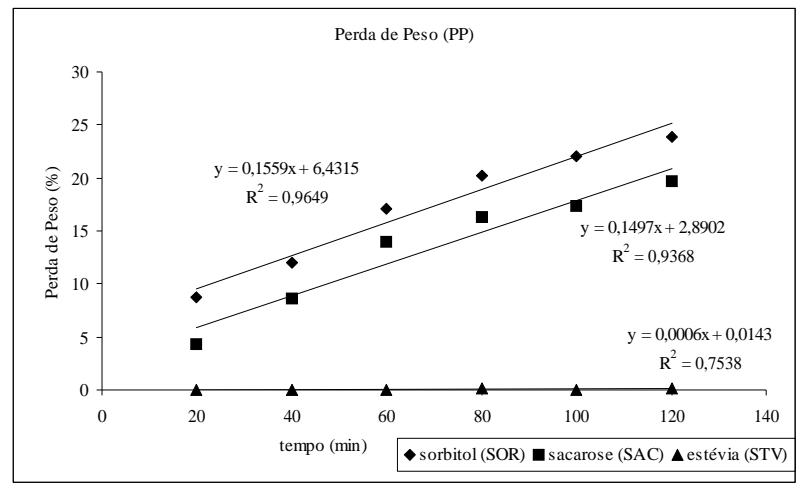

Comparando as figuras 01 e 02 observa-se que a perda de água é bem maior que o ganho de sólidos. Lenart (1996) e Tregunno \& Goff (1996) descreveram que quanto menor o ganho de sólidos num processo de desidratação osmótica melhor é o resultado em termos de cor, textura e sabor do produto final, pois esses se mantêm próximos ao sabor da fruta in natura. E quanto maior a perda de água, consequentemente menor será sua atividade de água e poderá ter um aumento em sua vida de prateleira, reduzindo o tempo do tratamento complementar.

Avaliando as figuras 01, 02 e 03 verifica-se que ocorreu uma maior perda de peso e perda de água para o tratamento com sorbitol, e um menor ganho de sólidos em relação ao tratamento com sacarose, esse fato foi relatado por Erba et al. (1994) que demonstraram que a presença de sorbitol promove uma diminuição da incorporação de sólidos e um aumento da perda de peso em pêssegos.

O sorbitol, apesar de ter uma maior facilidade de incorporação na fruta por causa do seu baixo peso molecular (PM=182), apresentou um menor ganho de sólidos (fig.02) em relação à sacarose, que conforme equação (02) leva em consideração a massa da fruta que está diretamente relacionada ao peso molecular do soluto e também à influência da perda de água (fig01) do mesmo, que é facilitada por solutos de baixo peso molecular.

\section{CONCLUSÃO}

O tratamento utilizando solução composta com estévia não se mostrou satisfatório por causa do baixo ganho de sólidos e reduzida perda de água na desidratação osmótica.

A maior perda de água $(33,46 \%)$ da manga durante a desidratação osmótica foi observada para o tratamento com sorbitol, enquanto o maior ganho de sólidos $(9,93 \%)$ foi obtido no tratamento com sacarose, uma maior perda de peso $(23,91 \%)$ também foi encontrada para a desidratação com sorbitol.

\section{AGRADECIMENTOS}

À CAPES pelo apoio financeiro.

\section{REFERÊNCIAS BIBLIOGRÁFICAS}

ANGELINI, R. Desidratação osmótica de kiwi (Actinidia deliciosa L.) estudo da reutilização da solução osmótica. 2002. Dissertação (Mestrado em Engenharia de Alimentos) - Universidade Estadual de Campinas, Campinas, 2002.

ANTONIO, G. C. Influência da estrutura celular e da geometria da amostra na taxa de transferência de massa do processo de desidratação osmótica de banana nanica (Musa cavendishi) e de mamão formosa (Carica papaya L.). 2002. 104 f. Dissertação (Mestrado em Engenharia de Alimentos) - Universidade Estadual de Campinas, Campinas, 2002.

ERBA, M. L.; FORNI, E.; COLONELLO, A.; GIANGIACOMO, R. Influence of sugar composition and air dehydration levels on the chemical-physical characteristics of osmodehydrofrozen fruit. Food Chemistry, Barkin, v. 50, n. 1, p. 69-73, 1994.

INSTITUTO ADOLFO LUTZ. Métodos químicos e físicos para análise de alimentos. São Paulo, 1985.

LENART, A. Osmo-convective drying of fruits and vegetables: technology and application. Drying technology, [S.1.], v. 14, p. 391-413, 1996.

LIMA, A. S.; FIGUEIREDO, R. W.; MAIA, G. A.; LIMA, J. R.; SOUZA NETO, M. A.; SOUZA, A. C. R. Estudo das variáveis de processo sobre a cinética de desidratação osmótica de melão. Ciência e Tecnologia de Alimentos, Campinas, v. 24, n. 2, abr./jun. 2004.

MACCARTHY, D. Concentration and drying of foods. London: Elsevier Applied Science, 1986. 
MIZRAHI, S.; EICHLER, S.; RAMON, O. Osmotic dehydration phenomena in gel systems. Journal of Food Engineering, Essex, v. 49, n. 2/3, p. 87-96, 2001.

SABLANI, S. S.; RAHMAN, M. S. Effect of syrupconcentration, temperature and sample geometry on equilibrium distribution coefficients during osmotic dehydration of mango. Food Research International, Barking, v. 36, p. 65-71, Mar. 2002.

SANTOS, C. N. P. Elaboração de um estruturado de polpa de manga (Mangifera indica L. cv Tommy Atkins) parcialmente desidratada por osmose. 2003. $79 \mathrm{f}$. Dissertação (Mestrado em Tecnologia de Alimentos) Universidade Estadual de Campinas, Campinas, 2003.

SOUZA NETO, M. A.; MAIA, G. A.; LIMA, J. R.; FIGUEIREDO, R. W.; SOUZA FILHO, M. de S. M.; LIMA,
A. S. Cinética de desidratação osmótica de manga. Publicatio UEPG - Ciências Exatas e da Terra, Ciências Agrárias e Engenharias, Ponta Grossa, v. 10, n. 2, p. 37 44, 2004.

TEDJO, W.; TAIWO, K. A.; ESHTIAGHI, M. N.; KNORR, D. Comparison of pretreatment methods on water and solid diffusion kinetics of osmotically dehydrated mangos. Journal of Food Engineering, Essex, n. 53, p. 133-142, 2002.

TORREGGIANI, D. Osmotic dehydration in fruit and vegetable processing. Food Research International, Barking, n. 26, p. 59-68, 1993.

TREGUNNO, N. B; GOFF, H. D. Osmodehydrofrezzing of apples: structural and textural effects. Food Research International, Barking, v. 29, p. 471-479, 1996. 